\title{
Conhecimento deficiente acerca do HIV/AIDS em estudantes adolescentes: identificação de diagnóstico de enfermagem da NANDA
}

\author{
Knowledge deficit regarding HIV/AIDS in adolescent students: identifying the NANDA nursing diagnosis
}

Conocimiento deficiente acerca de HIV/AIDS en estudiantes adolescentes: identificación de diagnóstico de enfermería de la NANDA

Jaqueline Galdino Albuquerque ${ }^{1}$, Patricia Neyva da Costa Pinheiro², Marcos Venícios Oliveira Lopes ${ }^{3}$, Maria de Fátima Antero Sousa Machado ${ }^{4}$

\section{RESUMO}

Este estudo objetivou identificar o diagnóstico de enfermagem - Conhecimento Deficiente acerca do HIV/AIDS - em adolescentes. Trata-se de um estudo transversal, desenvolvido em uma escola pública de Fortaleza-CE. A amostra foi composta por 182 estudantes, do sexo masculino, de 12 a 18 anos, regularmente matriculados. A coleta de dados aconteceu mediante um instrumento com assertivas sobre o HIV, para serem julgadas como verdadeiras ou falsas. As características definidoras do referido diagnóstico foram agrupadas conforme o desempenho do estudante na resolução das questões. O Conhecimento Deficiente esteve presente em mais de $\mathbf{9 0 \%}$ dos participantes, e a característica definidora mais frequente foi o Desempenho Inadequado em um Teste, conforme a Taxonomia da NANDA I. Diante do exposto, para os estudantes desta escola, as ações de enfermagem devem contemplar a utilização de estratégias educativas voltadas para prevenção, como o uso correto do preservativo masculino e formas de contaminação pelo HIV.

Descritores: Diagnóstico de Enfermagem; Conhecimento; Síndrome da Imunodeficiência Adquirida.

\section{ABSTRACT}

This study was performed with the objective to identify the nursing diagnosis - Knowledge deficit regarding HIV/AIDS - in adolescents. This cross-sectional study was performed at a public school located in Fortaleza - Ceará. The sample consisted of 182 male students, aged between 12 and 18 years, regularly enrolled at the referred school. The data collection was performed using an instrument containing statements regarding HIV, which the students marked as true or false. The defining characteristics of the referred diagnosis were grouped according to the student's performance regarding the instrument. Knowledge Deficit was present in more than $90 \%$ of the subjects, and the most frequent defining characteristic was Inadequate Performance on a Test, according to NANDA Taxonomy I. In light of these results, considering the referred students, nursing interventions should include educational strategies aimed at prevention, such as the correct use of condoms and the ways of becoming infected with HIV.

Descriptors: Nursing Diagnosis; Knowledge; Acquired Immune Deficiency Syndrome.

\section{RESUMEN}

Se objetivó identificar el diagnóstico de enfermería - Conocimiento deficiente acerca de HIV/AIDS- en adolescentes. Estudio transversal desarrollado en escuela pública de Fortaleza-Ceará. Muestra compuesta por 182 estudiantes de sexo masculino, de 12 a 18 años, regularmente matriculados. Colecta de datos realizada mediante instrumento con afirmaciones sobre HIV, a ser juzgadas como verdaderas o falsas. Las características definitorias del referido diagnóstico se agruparon de acuerdo al desempeño del estudiante en la resolución de las preguntas. El Conocimiento Deficiente se constató en más del $90 \%$ de los participantes, la característica definitoria más frecuente fue el Desempeño Inadecuado en un Test, conforme la Taxonomía de la NANDA I. Ante lo expuesto, para los estudiantes de esta escuela, las acciones de enfermería deben contemplar la utilización de estrategias educativas apuntando a la prevención y al uso correcto del preservativo masculino y formas de contagio del HIV.

Descriptores: Diagnóstico de Enfermería; Conocimiento; Síndrome de Inmunodeficiencia Adquirida.

\footnotetext{
1 Enfermeira, Mestre em Enfermagem, Professora Assistente, Universidade Federal de Pernambuco (UFPE). Vitoria de Santo Antão, PE, Brasil E-mail: jaquealbuquerque@terra.com.br.

2 Enfermeira. Doutora em Enfermagem, Professora Adjunta, Departamento de Enfermagem, Universidade Federal do Ceará (UFC). Fortaleza, CE, Brasil. E-mail: neyva.pinheiro@yahoo.com.br.

${ }^{3}$ Enfermeiro, Doutor em Enfermagem, Professor Adjunta, Departamento de Enfermagem, UFC. Fortaleza, CE, Brasil. E-mail: marcos@ufc.br.

4 Enfermeira, Doutora em Enfermagem, Professora Adjunto, Universidade Regional do Cariri. Crato, CE, Brasil. E-mail: fatimaantero@uol.com.br.
} 


\section{INTRODUÇÃO}

A adolescência é caracterizada por uma série de transformações biopsicossociais, que vão desde a maturação dos órgãos sexuais até a elaboração da identidade pessoal e sexual, com o exercício da sexualidade, intimidade e afetividade ${ }^{(1-2)}$. Além disso, nesta etapa do desenvolvimento humano, verifica-se uma variação no perfil da distribuição das doenças, identificando-se, mais comumente, um conjunto de agravos relacionados, sobretudo, com o comportamento sexual e as condutas que o adolescente apresenta dentro de seu convívio social(3).

Neste contexto, destacam-se as infecções sexualmente transmissíveis (IST), especialmente a AIDS com 592.914 casos diagnosticados no Brasil, no período de 1980 até junho de 2010, sendo 66.751 casos, acumulados nessas duas décadas, em pessoas na faixa etária de 13 a 24 anos. Desse total, 37.553 foram identificados entre jovens do sexo masculino e 29.196 do sexo feminino(4).

Esse número elevado, entre adolescentes e jovens, encontra-se associado a diversos fatores, dentre eles, a prática sexual desprotegida que os torna cada vez mais vulneráveis à aquisição de uma IST, em especial, a AIDS.

Contudo, essa noção de vulnerabilidade extrapola o aspecto relativo à conduta sexual apresentada por esses adolescentes, de tal modo que a forma de viver das pessoas, o grau e a qualidade de informação que estas apresentam sobre a AIDS e suas formas de transmissão e prevenção, bem como a capacidade de interpretarem tais informações e de incorporá-las como práticas preventivas são fatores a serem levados em consideração quanto se trata de vulnerabilidade ${ }^{(5)}$.

No entanto, embora se saiba da necessidade de inclusão do adolescente nesse processo, as práticas de saúde mostram-se excludentes, e o mesmo continua a apresentar conhecimento superficial quanto às IST, bem como comportamentos de risco para a aquisição das mesmas, em especial, a contaminação pelo HIV.

Os estudos ${ }^{(6-8)}$ sobre 0 conhecimento dos adolescentes acerca das IST/AIDS vêm sendo explorados em várias circunstâncias, contudo, o enfoque voltado para o uso de uma terminologia própria da enfermagem, não tem sido frequente. Além disso, o enfermeiro não tem explorado de maneira sistemática o ambiente escolar onde o adolescente passa grande parte do seu tempo participando de atividades.
A importância da escola tem sido enfatizada como um espaço de socialização, em que o diálogo entre os amigos e professores pode ser explorado para tratar de aspectos relacionados com as IST, especialmente a AIDS, desenvolvendo práticas de promoção da saúde dentro desse contexto ${ }^{(9)}$.

Reforçando essa idéia, a educação em saúde, reveste-se de singular importância no processo de promoção da saúde dentro do âmbito escolar, pois está relacionada com a aprendizagem construída para alcançar a saúde, devendo, necessariamente, voltar-se ao atendimento da população, dentro do contexto onde está inserida ${ }^{(10)}$.

$O$ enfermeiro insere-se nesse contexto como profissional que possui habilidades e competências específicas incluindo desde a promoção da saúde, até a reabilitação dos usuários. No entanto, algumas reflexões importantes devem ser consideradas quanto a conduta do enfermeiro no processo de educação em saúde, que tem sido baseada no repasse de informações, sem participação ativa dos sujeitos e pouco estímulo à reflexão e construção do pensamento crítico. Esta visão também é observada na prática de outros profissionais da saúde, nas relações que estabelecem com os usuários e familiares, desconsiderando a multidimensionalidade deste processo ${ }^{(11) .}$

Aprofundou-se, nesta pesquisa, a investigação sobre o diagnóstico de enfermagem Conhecimento Deficiente dos adolescentes acerca do HIV/AIDS. A taxonomia II da NANDA apresenta, em seu domínio Percepção/Cognição, o diagnóstico Conhecimento Deficiente, e o define como sendo a "ausência ou deficiência de informação cognitiva relacionada a um tópico específico"(12).

Estudar esse diagnóstico em adolescentes possibilitará a construção de meios de intervenção adequados a essa realidade, e as ações de enfermagem mostrar-se-ão mais direcionadas e voltadas para as necessidades desta clientela.

Diante do exposto, objetivou-se neste trabalho analisar o diagnóstico de enfermagem "Conhecimento deficiente acerca do HIV/AIDS" em adolescentes do sexo masculino, de uma escola pública em Fortaleza-Ceará.

\section{MATERIAL E MÉTODOS}

Trata-se de um estudo transversal de abordagem quantitativa. Os estudos transversais são aqueles realizados em um único momento, sem período de 
acompanhamento, sendo úteis quando se quer descrever variáveis e seus padrões de distribuição(13).

A investigação foi realizada em uma instituição pertencente à rede pública de ensino, localizada em um bairro da periferia da cidade de Fortaleza-CE. A seleção da escola teve como critério a não realização de atividades educativas referentes ao HIV/AIDS, no ano de 2008, pois estas poderiam influenciar as respostas dos estudantes, bem como a identificação das características definidoras do diagnóstico em estudo.

A escola foi fundada há 45 anos e, atualmente, funciona nos três turnos, com 51 professores e 22 funcionários, com capacidade média de 1300 alunos por ano. Além disso, desenvolve alguns projetos, tais como atividades esportivas, informática, hora da leitura, na qual os alunos usufruem do espaço da biblioteca para suas atividades, e parcerias com outras instituições para oferta de cursos profissionalizantes com encaminhamento ao primeiro emprego.

A opção de desenvolver esta pesquisa no âmbito escolar surgiu em virtude da experiência da pesquisadora com atividades de extensão, nas quais se utilizavam estratégias educativas voltadas para os temas relativos às infecções sexualmente transmissíveis.

A população foi composta por adolescentes do sexo masculino, com idade de 12 a 18 anos, fase da adolescência, conforme o Estatuto da Criança e do Adolescente ${ }^{(14)}$, regularmente matriculados nas escolas. Dos 1220 alunos matriculados, 547 pertenciam ao sexo masculino, compondo a população do estudo.

Como critérios de inclusão foram definidos: estudantes do sexo masculino que estivessem na faixa etária de 12 a 18 anos. Os critérios de exclusão considerados foram: não aceitação por parte do adolescente, visto que a participação era voluntária; não autorização dos pais ou responsável; participantes que solicitaram o auxílio dos colegas para a resolução das assertivas, embora tenham sido previamente orientados quanto a isso, e/ou conversaram excessivamente durante a resolução das questões, permanecendo desatentos na maior parte do tempo.

Convém salientar que os instrumentos incompletos, ilegíveis e preenchidos erroneamente foram excluídos na etapa de análise dos dados.

Após a investigação desses critérios, obteve-se uma amostra de 182 estudantes. A grande perda de participantes aconteceu em virtude dos seguintes fatores: ausência de muitos estudantes no período de coleta de dados que ocorreu nos meses de maio e junho de 2008; não devolução dos termos de consentimento devidamente assinados pelos pais ou responsável; e a localização da escola em uma área de tráfico e consumo de drogas que dificultou a coleta durante o turno da noite.

O instrumento de coleta de dados utilizado foi um questionário auto-aplicável subdividido em duas sessões: 1. Dados de identificação com as variáveis: idade, escolaridade, ocupação, estado civil, renda familiar, número de membros da família, etilismo, tabagismo e uso de drogas; 2. Dados referentes às características definidoras e aos fatores relacionados do diagnóstico Conhecimento deficiente acerca do HIV/AIDS.

As características definidoras selecionadas para este estudo foram: desempenho inadequado em um teste; seguimento inadequado de instruções; e verbalização do problema. As demais características, comportamentos impróprios e comportamentos exagerados, foram excluídos, pois necessitam de estudos com outras abordagens e exigem períodos de acompanhamento, não se enquadrando, portanto nas dimensões desta pesquisa.

Para a identificação das características definidoras, estabeleceram-se os seguintes indicadores:

- Desempenho inadequado em um teste foi avaliado por meio da percentagem de acertos nas questões pertinentes às formas de prevenção e contaminação pelo HIV, assim como ao uso correto do preservativo masculino. Se, em pelo menos uma destas questões, o participante apresentou uma percentagem de acertos inferior a $80 \%$, considerou-se a característica como presente;

- Seguimento inadequado de instruções esteve presente quando a percentagem de acertos da questão relativa ao uso correto do preservativo masculino foi menor do que $80 \%$;

- Verbalização do problema foi identificada quando o participante relatou não apresentar conhecimento acerca das temáticas abordadas nos questionamentos.

A percentagem de $80 \%$ utilizada nas duas primeiras características foi definida com base no manual de formação de formadores que o tem como o parâmetro para o julgamento da necessidade de informações 
adicionais, ou não, com relação a um determinado tópico. Convém salientar que este percentual aplica-se apenas no caso de não terem sido utilizadas exposições anteriores sobre uma temática, neste caso, HIV/AIDS ${ }^{(15)}$. Isto justifica o critério de seleção das escolas quanto a não realização de atividades referentes à temática em estudo.

Os fatores relacionados selecionados foram: falta de exposição; falta de familiaridade com os recursos de informação; e falta de interesse em aprender.

Para a sua identificação, estabeleceram-se os seguintes indicadores:

- Falta de exposição foi avaliada a partir das exposições, ativa e passiva, às informações sobre o HIV/AIDS, relativas aos aspectos gerais sobre o vírus, bem como às formas de transmissão, medidas de prevenção e uso correto do preservativo masculino.

- Falta de familiaridade com recursos de informação foi definida através de questões que se referiram aos meios onde o adolescente pode obter informações corretas sobre o HIV/AIDS. Se em pelo menos uma das questões acima o adolescente assinalou menos de $80 \%$ das opções apresentadas, considerou-se fator relacionado como presente.

- Falta de interesse em aprender foi definida como presente quando o adolescente relatasse não apresentar interesse em buscar informações, ou não dar importância à temática em estudo.

Antes da aplicação, o instrumento foi submetido a três especialistas na área de saúde do adolescente e HIV/AIDS, para avaliação de aparência e conteúdo do mesmo, sendo feitas às devidas correções sugeridas.

Realizou-se, ainda, um estudo piloto com 14 adolescentes para que os mesmos avaliassem o instrumento quanto a sua aplicabilidade, dúvidas e tempo de preenchimento.

Os dados foram coletados no período de junho a julho de 2008. Os adolescentes foram previamente esclarecidos sobre os objetivos da pesquisa e o preenchimento do questionário. O pesquisador manteve-se presente em todo o procedimento de coleta, esclarecendo dúvidas quando necessário.

As questões cujas respostas eram do tipo "verdadeiro" ou "falso" foram corrigidas conforme o parâmetro estabelecido de $80 \%$. Com essa avaliação, identificaram-se as características definidoras, definiu-se a existência ou não do diagnóstico Conhecimento Deficiente, e posteriormente, dos fatores relacionados.

Para a análise, os dados foram armazenados em uma planilha no Excel 2003, e analisados com apoio do software SPSS versão 17.0.

Os dados foram apresentados em tabelas, com as respectivas estatísticas descritivas e valores de testes utilizados.

O projeto foi encaminhado ao Comitê de Ética da Universidade Federal do Ceará, obtendo parecer favorável, com o número de protocolo 112/08. Atendeuse aos aspectos contidos na Resolução 196/96 sobre pesquisa com seres humanos do Conselho Nacional de Saúde/Ministério da Saúde ${ }^{(16)}$.

Além disso, foram garantidos o anonimato do adolescente e a liberdade de participar ou não do estudo. Também foi assegurado que a pesquisa não traria nenhum prejuízo nas atividades escolares. O participante foi esclarecido sobre todos os objetivos da mesma e, no caso de não maioridade, os pais ou responsáveis assinaram a autorização para a participação do adolescente no estudo, através do Termo de Consentimento Livre e Esclarecido.

\section{RESULTADOS E DISCUSSÃO}

Quanto à caracterização da amostra, observou-se que aproximadamente $70,0 \%$ dos adolescentes situaram-se na faixa etária de 15 a 18, com média de 15 anos, e 14,3\% desenvolviam alguma atividade trabalhista, além de estudarem. Cerca de 60,0\% cursavam séries do ensino médio.

Os indicadores utilizados para a identificação das características definidoras do diagnóstico em estudo estão apresentados na Tabela 1. 
Tabela 1: Distribuição dos adolescentes, de acordo com o desempenho inferior à $80,0 \%$, quanto aos indicadores de conhecimento sobre o HIV, suas formas de contaminação e prevenção, bem como o uso correto do preservativo masculino. Fortaleza, CE, 2008.

\begin{tabular}{ccc}
\hline Variáveis & \multicolumn{2}{c}{ Desempenho } \\
\cline { 2 - 3 } & $\mathbf{N}^{\circ}$ & $\%$ \\
\hline Indicador 1: Conhecimento sobre o HIV/AIDS & 135 & 74,1 \\
Indicador 2: Conhecimento sobre as formas de contaminação do HIV/AIDS & 142 & 78,0 \\
Indicador 3: Conhecimento sobre o uso correto do preservativo masculino & 94 & 51,6 \\
Indicador 4: Conhecimento sobre as formas de prevenção contra o HIV/AIDS. & 127 & 69,7 \\
\hline
\end{tabular}

Os resultados apresentados se aproximam dos achados de um estudo com adolescentes de escolas públicas e particulares ${ }^{(14)}$, em que se viu que a instituição pública obteve média de idade de 15,1 anos. Contudo, outros autores encontraram resultados diferentes, visto que a média de idade para os alunos da escola pública foi de 17,4 , e com relação à ocupação, observou-se que aproximadamente a metade dos participantes declarou ter experiência de trabalho remunerado, onde a maioria pertenceu à escola pública(7).

Observa-se que mais de $50,0 \%$ dos adolescentes apresentaram um desempenho inferior ao parâmetro estabelecido no que se diz respeito ao uso correto do preservativo masculino.

Para as formas de prevenção, o percentual foi de $69,7 \%$. O conhecimento sobre informações gerais do HIV/AIDS apresentou $74,1 \%$ de estudantes com desempenho inferior a $80,0 \%$ e, finalmente, $78,0 \%$ não atingiu o número de acertos esperado quando avaliados acerca das formas de contaminação pelo vírus da AIDS.

Um estudo sobre conhecimentos e práticas de adolescentes evidenciou que $94,5 \%$ relataram conhecer o preservativo como método eficaz ${ }^{(8)}$. No entanto, o fato de se considerarem conhecedores do método, não se traduz em uma prática sistemática.

Embora os adolescentes afirmem que a camisinha é o método mais conhecido entre eles, o seu uso tem-se dado de modo incorreto e irregular, demonstrando que considerar-se bem informado não significa, de fato, que essa informação é a mais adequada e, portanto, necessita de intervenções que esclareçam essas lacunas existentes $^{(17)}$.

Na Tabela 2 encontram-se as estatísticas descritivas dos indicadores supracitados.

Tabela 2: Estatísticas descritivas dos indicadores de conhecimento sobre o HIV, suas formas de contaminação e prevenção, bem como o uso correto do preservativo masculino. Fortaleza, CE, 2008.

\begin{tabular}{lcccc}
\hline \multicolumn{1}{c}{ Variáveis } & Média & Mediana & D.P & K-S (p) \\
\hline Indicador 1: Conhecimento sobre o HIV/AIDS & 14,70 & 15,00 & 2,54 & 0,040 \\
$\begin{array}{l}\text { Indicador 2: Conhecimento sobre as formas de contaminação do } \\
\text { HIV/AIDS }\end{array}$ & 16,49 & 17,00 & 2,85 & 0,026 \\
$\begin{array}{l}\text { Indicador 3: Conhecimento sobre o uso correto do preservativo } \\
\text { masculino }\end{array}$ & 12,15 & 12,00 & 2,06 & 0,001 \\
$\begin{array}{l}\text { Indicador 4: Conhecimento sobre as formas de prevenção contra o } \\
\text { HIV/AIDS. }\end{array}$ & 4,47 & 5,00 & 0,84 & 0,000 \\
\hline
\end{tabular}

D.P: desvio-padrão; K-S: Teste de Kolmogorov-Smirnov.

A média de acertos do primeiro indicador foi 14,70 assertivas. Quanto ao conhecimento sobre as formas de contaminação e de prevenção viu-se uma média de 16,49 e 4,47 itens, respectivamente. E com relação ao uso correto do preservativo masculino, encontrou-se uma média de 12,15 alternativas corretas.

O teste de Kolmogorov-Smirnov, que avalia a normalidade dos dados, evidenciou distribuição assimétrica nos quatro indicadores citados conforme o valor $p<0,05$. Esse teste é utilizado para verificar se existem diferenças entre os escores identificados na amostra (número de acertos) e os escores de alguma distribuição especificada, neste caso, distribuição normal. Diante disso, para o raciocínio dos testes de normalidade, com o valor $\mathrm{p}<0,05$ observa-se que existem diferenças entre o que foi observado e o que era esperado tratando-se, portanto, de uma distribuição não-paramétrica.

É possível que os indicadores utilizados necessitem de ajustes quanto aos seus conteúdos e adaptação específica à clientela estudada, levando em considerações suas peculiaridades e demandas, fato que 
pode justificar a assimetria da distribuição de dados referentes à variável desempenho nos testes.

A apresentação dos resultados relativos ao diagnóstico de enfermagem Conhecimento Deficiente acerca do HIV/AIDS, suas características definidoras e fatores relacionados estão contidos na Tabela 3.

Tabela 3: Distribuição das características definidoras, fatores relacionados e do diagnóstico de enfermagem Conhecimento Deficiente acerca do HIV/AIDS, em adolescentes de uma escola pública. Fortaleza, CE, 2008.

\begin{tabular}{|c|c|c|c|c|}
\hline \multirow{2}{*}{ Variáveis } & \multicolumn{2}{|c|}{ Presente } & \multicolumn{2}{|c|}{ Ausente } \\
\hline & No & $\%$ & $\mathbf{N}^{\circ}$ & $\%$ \\
\hline \multicolumn{5}{|l|}{ Diagnóstico de Enfermagem } \\
\hline Conhecimento Deficiente acerca do HIV/AIDS & 181 & 99,4 & 1 & 0,6 \\
\hline \multicolumn{5}{|l|}{ Características Definidoras } \\
\hline Desempenho inadequado em um teste & 181 & 99,4 & 1 & 0,6 \\
\hline Seguimento inadequado de instruções & 94 & 51,6 & 88 & 48,4 \\
\hline Verbalização do Problema & 36 & 19,7 & 146 & 80,3 \\
\hline \multicolumn{5}{|l|}{ Fatores Relacionados } \\
\hline Falta de familiaridade com os recursos de informação & 145 & 79,6 & 37 & 20,4 \\
\hline Falta de exposição & 57 & 31,3 & 125 & 68,7 \\
\hline Falta de interesse em aprender & 33 & 18,1 & 149 & 81,9 \\
\hline
\end{tabular}

O diagnóstico em estudo foi identificado em quase $100,0 \%$ dos participantes da pesquisa, sendo a característica definidora - desempenho inadequado em um teste - presente em 99,4\%, a que determinou claramente a presença dessa resposta humana. Tal característica estaria presente nos estudantes que não atingissem o número de acertos estabelecido (parâmetro de 80,0\%). O indicador cujo desempenho mostrou-se mais comprometido esteve relacionado com o conhecimento sobre as formas de contaminação do HIV, enquanto cerca de 50,0\% apresentaram um bom desempenho na resolução de questões relativas ao uso correto do preservativo masculino, que avaliou a característica definidora - seguimento inadequado de instruções - identificada em um pouco mais da metade dos adolescentes.

Em um estudo já citado(6), observou-se que alunos de escolas públicas e privadas apresentaram conhecimento adequado sobre a camisinha masculina, cujo quantitativo de acertos foi superior a $70,0 \%$. Em nosso estudo, esse percentual foi de $50,0 \%$.

A verbalização da ausência de conhecimento sobre o HIV/AIDS não foi uma característica marcante na clientela estudada, visto que apenas $19,7 \%$ referiram não possuir informação sobre a temática questionada. $O$ fato acima mostra que os adolescentes já receberam algum conhecimento sobre HIV/AIDS, no entanto o impacto que este tem causado na sua tomada de decisão não parece ser tão perceptível, a ponto de mudar seus comportamentos.

No Brasil, observa-se uma série de campanhas de prevenção às IST, com o estímulo ao uso do preservativo masculino. No entanto, o simples repasse de informações de modo verticalizado, sem reflexão crítica e participação ativa do adolescente nesse processo, tem dissociado a informação da adoção de práticas preventivas $^{(8)}$.

Pesquisas sobre essa temática têm mostrado que os adolescentes possuem informações sobre a prevenção das IST, contudo estas são insuficientes para promover mudanças nos comportamentos e condutas, por diversos fatores, tais como a qualidade duvidosa e a restrita acessibilidade dessa informação, que se mostra escassa e superficial, sendo assim, o simples fato de se ter conhecimento sobre essas enfermidades não implica necessariamente na adoção de medidas preventivas ${ }^{(8,17)}$.

Quanto aos fatores relacionados, verificou-se a falta de familiaridade com os recursos da informação em 145 participantes $(79,6 \%)$. A falta de exposição e a falta de interesse em aprender foram identificadas em 57 participantes (31,3\%) e 33 estudantes (18,1\%), respectivamente.

A falta de familiaridade com os recursos da informação refere-se à quantidade e qualidade desses recursos para fornecimento de dados sobre o tema em estudo, na ótica dos adolescentes. 
Os meios mais citados como adequados para se obter conhecimento foram, nesta ordem, os profissionais de saúde, os professores e a escola, sendo os amigos os menos apontados. Contudo, os adolescentes não têm a conduta periódica de buscar esses profissionais de saúde para discutir o tema, sendo a internet, os professores (em diálogos informais) e os pais os mais procurados.

Com o advento das redes sociais, a juventude tem utilizado a internet rotineiramente, possibilitando o diálogo entre pessoas da mesma idade para falar sobre diversos assuntos, tais como relações afetivas, incluindo o sexo, envolvendo também o tema relativo às ISTs. Portanto, o que se observa é que essa ferramenta de comunicação pode não somente ser uma fonte de informação, mas também de troca de experiências entre esses adolescentes.

Porém, um estudo recente mostrou que o uso dessas redes e da internet pode estimular ou não os comportamentos de risco entre esse público. Além disso, podem incrementar o conhecimento sobre prevenção. $O$ que vai determinar a capacidade da ferramenta em desempenhar esses papéis é a forma como ela é utilizada $^{(18)}$.

Diante disso, sugere-se que os profissionais de saúde passem a incluir essas redes sociais em suas estratégias educativas, criando tópicos de discussão sobre o assunto, envolvendo a sexualidade de forma ampla, desde os comportamentos saudáveis até o conhecimento sobre as patologias. O adolescente precisa ser um participante ativo no processo de ensinoaprendizagem, com abordagem horizontalizada, levando em consideração seus conceitos, valores, culturalidade e comportamentos já aprendidos.

Observa-se uma forte necessidade de se extrapolar o aspecto meramente informativo que tem permeado as ações educativas no âmbito da atenção à saúde do adolescente, com o intuito de possibilitar ao sujeito as escolhas responsáveis acerca de sua sexualidade, de forma consciente ${ }^{(19)}$.

A média de recursos utilizados foi de 2,77 por estudante, numa lista de 10 opções (internet, meios de telecomunicação/revistas, amigos, pais, profissionais de saúde, professores, palestras/cursos, igrejas, hospitais e escolas).

A escola foi um dos meios menos procurados pelos participantes, embora seja listada como uma entidade informativa, conforme citado anteriormente. Convém salientar que na escola onde foi desenvolvida esta pesquisa não havia nenhum tipo de atividade, ainda que somente de repasse de informações. Esse fato é de fundamental importância, uma vez que o espaço escolar proporciona ao estudante adolescente um mundo de ideias, criatividade e descobertas cruciais para a formação da cidadania do sujeito. No entanto, essa função não foi observada como estratégia dessa escola.

Faz-se necessária uma maior compreensão das dificuldades enfrentadas pelos professores e demais profissionais da escola em abordar a temática com os estudantes, a fim de desmistificar temas, preconceitos, e conhecer as percepções de todos os envolvidos nesse processo, para que a instituição educadora possa, de fato, educar e proporcionar a construção de conceitos e valores, bem como estimular a formação do pensamento crítico-reflexivo nesses estudantes ${ }^{(8)}$.

\section{CONCLUSÕES}

O diagnóstico Conhecimento Deficiente foi identificado na grande maioria dos estudantes, e a característica definidora mais frequente foi $o$ desempenho inadequado em um teste. Os indicadores cujos desempenhos mostraram-se mais comprometidos estiveram relacionados, respectivamente, com o conhecimento sobre as formas de contaminação do HIV e uso correto do preservativo masculino, que avaliou a característica definidora - seguimento inadequado de instruções - identificada em um pouco mais da metade dos adolescentes. Para os fatores relacionados, a falta de familiaridade com os recursos da informação esteve presente em uma considerável parcela de estudantes.

O reduzido número de investigações sobre esse diagnóstico de enfermagem em clientelas semelhantes foram alguns dos fatores que dificultaram o percurso deste estudo, em especial a etapa de construção dos indicadores.

Portanto, sugere-se a realização de pesquisas voltadas para a validação acurada dessa resposta humana, possibilitando a aplicação de intervenções de enfermagem específicas para o adolescente, respeitando todas as suas potencialidades, vendo-o de modo individual e coletivo, como um sujeito e não como um ser passivo.

O enfermeiro enquanto membro da equipe multiprofissional exerce um papel de fundamental 
importância quanto à promoção da saúde do adolescente, uma vez que possui formação acadêmica e profissional para desempenhar a função de educador em saúde, seja no âmbito hospitalar, da atenção primária e escolar, sendo esse último um local de grandes oportunidades para se abordar os mais diversos temas que envolvem o universo do adolescente, e que extrapolam as ações específicas do setor saúde, necessitando de estratégias interdisciplinares que possam ser resolutivas às demandas biopsicossociais do adolescente.

14. Ministério da Saúde. Estatuto da Criança e do Adolescente. Série E. Legislação de Saúde. 3 ed. Brasília (Brasil): Ministério da Saúde. 2006. 96p.

15. Bossemeyer D, Moura ERF. Formação de formadores: manual de referência (revisão e adaptação para o Programa de Apoio à Prevenção do HIV/SIDA). 2. ed. Baltimore: JHPIEGO/ Johns Hopkins University, 2006.

16. Ministério da Saúde; Conselho Nacional de Saúde. Resolução No 196/96 - Normas regulamentadoras de pesquisa envolvendo seres humanos. Brasília (Brasil): Ministério da Saúde; 1996.

17. Dias FLA, Silva KL, Vieira NFC, Pinheiro PNC, Maia CC. Riscos e vulnerabilidades relacionadas à sexualidade na adolescência. Rev. enferm. UERJ. 2010;18(3):456-61. 18. Young SD, Rice E. Online Social Networking Technologies, HIV Knowledge, and Sexual Risk and Testing Behaviors Among Homeless Youth. AIDS Behav. 2011;15(2):253-60.

19. Carmo R, Van der Sand ICP. O discurso dos adolescentes sobre vida sexual na adolescência. Revista Eletrônica de Enfermagem [Internet]. 2007 [cited 2011 nov 05];9(2):41731. Available from: http://www.fen.ufg.br/revista/v9/n2/v9n2a10.htm.

Artigo recebido em 26.10.2010. Aprovado para publicação em 13.03.2012. Artigo publicado em 30.03.2012. preservativo masculino e ao conhecimento sobre DST/AIDS em adolescentes de escolas públicas e privadas do Município de São Paulo, Brasil. Cad. Saude Publica. 2006;22(2):31523.

7. Camargo BV, Botelho LJ. Aids, sexualidade e atitudes de adolescentes sobre proteção contra o HIV. Rev. Saude Publica. 2007;41(1):1-8.

8. Oliveira DC de, Pontes APM de, Gomes AMT, Ribeiro MCM. Conhecimentos e práticas de adolescentes acerca das DST/HIV/AIDS em duas escolas públicas municipais do Rio de Janeiro. Esc Anna Nery Rev Enferm.. 2009;13(4):833-41. 9. Beserra EP, Torres CA, Pinheiro PNC, Alves MDS, Barroso MGT. Pedagogia freireana como método de prevenção de doenças. Ciência \& Saúde Coletiva. 2011;16(Supl.1):15631570 . 10. Oliveira HM de, Gonçalves MJF. Educação em Saúde: uma experiência transformadora. Rev. bras. Enferm. 2004;57(6):761-63.

11. Backes VMS, Lino MM, Prado ML do, Reibnitz KS, Canaver BP. Competência dos enfermeiros na atuação como educador em saúde. Rev. bras. enferm.. 2008;61(6):858-65. 12. North American Nursing Diagnosis Association International (NANDA International). Diagnósticos de enfermagem da NANDA: definições e classificações 20092011. Porto Alegre: Artmed, 2010. 198p. 13. Hulley SB, Cummings SR, Browner WS, Grady DG, Newman TB. Delineando a pesquisa clínica: uma abordagem epidemiológica. 2. ed. Porto Alegre: Artmed, 2008. 